\title{
FINANCIAL TIME SERIES FORECASTING USING FUZZY REARRANGED INTERVALS
}

\author{
Hye-Young Jung ${ }^{a}$, Jin Hee Yoon ${ }^{b}$ And Seung Hoe Choi ${ }^{c, *}$
}

\begin{abstract}
The fuzzy time series is introduced by Song and Chissom([8]) to construct a pattern for time series with vague or linguistic value. Many methods using the interval and fuzzy logical relationship related with historical data have been suggested to enhance the forecasting accuracy. But they do not fully reflect the fluctuation of historical data. Therefore, we propose the interval rearranged method to reflect the fluctuation of historical data and to improve the forecasting accuracy of fuzzy time series. Using the well-known enrollment, the proposed method is discussed and the forecasting accuracy is evaluated. Empirical studies show that the proposed method in forecasting accuracy is superior to existing methods and it fully reflects the fluctuation of historical data.
\end{abstract}

\section{INTRODUCTION}

Time series analysis has been a very popular method with many successful applications. In time series analysis, the involved experimental data are assumed to be precise. However, in many practical situations we encounter data which are not only random but vague due to ambiguous information or linguistic structure as well, for example 'about 10', 'greater than 10', 'more or less between 5 and 10', or 'fair', 'good', 'excellent', etc. Moreover, sometimes in the context of economic systems, such as stock market price, foreign exchange rate or market sales price of particular commodities, the historical data record officially, closing values, rough average values, maximum values or minimum values, instead of the precise series with variation during the time period. And usually we use only closing values when we analyze financial daily data. In these situations, sometimes closing values cannot be the representative value of one day, especially when closing value is the highest or lowest value of the day. Also, if we use only closing value for data analysis, we might lose

Received by the editors November 11, 2011. Revised January 13, 2012. Accepted Jan. 26, 2012. 2000 Mathematics Subject Classification. 37M10, 62M10, 62P20, 91B84.

Key words and phrases. fuzzy time series, forecasting, rearranged interval method, fluctuation.

${ }^{*}$ Corresponding author. 
lots of data information of the day. So, it is better for us to use fuzzy data which include variation of the day than using general time series models such as ARIMA, $\mathrm{ARCH}$ and GARCH in these cases. Furthermore, if the number of observed data is fewer than 50, it is not valid to apply the general time series models. An appropriate way of solving such problems is by the use of fuzzy time series model using the concept of fuzzy set.

The fuzzy time series was introduced by Song and Chissom([8]) to forecast the enrollments of the University of Alabama. Since then, the new methods of the fuzzy time series to reduce the forecasting error have been studied by many authors. Since the fuzzy time series is a forecasting method, reducing the forecasting error is very important. Chen $([1])$ proposed a method for forecasting the enrollments of the University of Alabama based on the simplified arithmetic operations. Huarng([3]) proposed a heuristic model for forecasting the enrollments of the University of Alabama and the Taiwan Futures Exchange(TAIFEX) based on heuristic knowledge. In [7], Liu suggested a improved fuzzy time series model with a trapezoidal fuzzy number based on the Chen's method. Lee et al.([6]) proposed a model for forecasting the temperature and the TAIFEX using the high-order fuzzy logical relationships and genetic simulated annealing techniques. Cheng et al.([2]) applied a fuzzy time series model based on the adaptive expectation model for forecasting the TAIEX and the enrollments of the University of Alabama.

However, the models mentioned above have some drawbacks: first, the heuristic model requires an assumption that there are heuristic knowledge showing the increase or decrease in historical data for the next period or the other variables to forecast the historical data. Second, high-order model is not easy to calculate. Third, the models mentioned above do not fully reflect the fluctuation of the historical data.

In this paper, we propose a new method to resolve these drawbacks. First, our method provides an easy calculation and does not need heuristic knowledge or other variables. Second, to better reflect the fluctuation of historical data, we consider the frequency of historical data in each interval and variation of fuzzy logical relationship. If the interval has the high frequency of historical data then the fuzzy time series model does not reflect the fluctuation of historical data well. Also, if forecasts have the same values even though the historical data have a great difference, then the forecasting accuracy is reduced. Thus, our method based on the frequency of historical data in the interval and variation in the fuzzy logical 
relationship group would provide better the forecasting accuracy and reflect actual data's fluctuation better than the existing methods.

The rest of this paper is organized as follows. In Section 2, we review some definitions for fuzzy time series. In Section 3, our new method is introduced. In Section 4, we present our new method to forecast the enrollments of the University of Alabama and TAIFEX. Also, we introduce the new target data KRW/USD average exchange rate per month(KUEXR). In Section 5, compares the forecasting accuracy with the previous methods' results. The conclusions are discussed in Section 6 .

\section{Fuzzy Time Series}

In this section, we give some definitions for fuzzy time series proposed by Song and Chissom.

Let $U=\left\{u_{1}, u_{2}, \cdots, u_{n}\right\}$ be the universe of discourse containing all values of a time series. Define a fuzzy set $A_{i}$ of $U$ :

$$
A_{i}=\mu_{A_{i}}\left(u_{1}\right) / u_{1}+\mu_{A_{i}}\left(u_{2}\right) / u_{2}+\cdots+\mu_{A_{i}}\left(u_{n}\right) / u_{n},
$$

where $\mu_{A_{i}}$ is the membership function of fuzzy set $A_{i}$ and $1 \leq i \leq n$.

Definition 1. If $F(t)$ consists of fuzzy set $A_{i}$, then $\{F(t): t=\cdots, 0,1,2, \cdots\}$ is called a fuzzy time series.

Definition 2. Let $F(t)$ be a fuzzy time series. If there exists a fuzzy relationship $R(t, t-1)$ such that $F(t)=F(t-1) \circ R(t, t-1)$ where $\circ$ represents an fuzzy operator, then $F(t)$ is said to be caused by $F(t-1)$.

Definition 3. Assume that $F(t)$ is a fuzzy time series and $F(t)$ is caused by $F(t-1), F(t-2), \cdots$, and $F(t-n)$. Then $F(t)$ is called the $n$-th order fuzzy time series.

Definition 4. When $F(t-1)=A_{i}$ and $F(t)=A_{j}$, the fuzzy logical relationship between $F(t-1)$ and $F(t)$ is denoted by $A_{i} \rightarrow A_{j}$. The fuzzy logical relationship " $A_{i} \rightarrow A_{j}$ " denotes that if the fuzzified historical data of time $t-1$ is $A_{i}$, then the fuzzified historical data of time $t$ is $A_{j}$. Note that the repeated fuzzy logical relationships are removed ([1]).

Definition 5. Fuzzy logical relationships can be grouped together into fuzzy logical relationship groups. Suppose there are fuzzy logical relationships such that : $A_{i} \rightarrow$ $A_{j 1}, A_{i} \rightarrow A_{j 2}, \cdots$, and $A_{i} \rightarrow A_{j k}$. Then the fuzzy logical relationship group is denoted by $A_{i} \rightarrow A_{j 1}, A_{j 2}, \cdots, A_{j k}$. 


\section{Fuzzy Time Series Using Rearranged Intervals}

In this section, we propose a new method to improve forecasting accuracy, based on partitioning the intervals and fuzzy relationships. The algorithm for the proposed method is as follows.

Step 1: Define the universe of discourse $U$.

Let $x_{(1)}$ and $x_{(n)}$ be the maximum and the minimum value of historical data $\left\{x_{t}\right.$ : $t=1,2, \cdots, n\}$. Then the universe of discourse $U$ is defined by $U=\left[x_{(1)}-c, x_{(n)}+d\right]$, where $c$ and $d$ are two proper positive numbers.

Step 2: Partition the universe of discourse into several intervals.

We consider a method splitting up the universe set using the intervals. First, we use the intervals $v_{j}=\left[e_{j-1}, e_{j}\right]\left(j=1, \cdots, m_{1}\right)$ with equal length $(l)$ to divide the set $U$ into some subsets. Here,

$$
e_{j}=\left\{\begin{aligned}
x_{(1)}-c & \text { if } j=0 \\
x_{(1)}-c+j \cdot l & \text { if } 1 \leq j \leq m_{1}-1, \\
x_{(n)}+d & \text { if } j=m_{1} .
\end{aligned}\right.
$$

Next, if the historical data $x_{t}$ is an left endpoint of the interval $v_{j+1}$, we divide the union of two interval $v_{j}$ and $v_{j+1}$ into several intervals $v_{j, j+1}^{(i)}\left(i=1, \cdots, m_{2}\right)$ satisfying the following conditions:

(i) Both intervals $v_{j, j+1}^{(i)}=\left[e_{j, j+1}^{(i-1)}, e_{j, j+1}^{(i)}\right]$ has same length $\left(l_{1}\right)$ and the historical data $x_{t}$ is not an endpoint of the interval $v_{j, j+1}^{(i)}$ for each $i$.

(ii) The endpoint $e_{j, j+1}^{(i)}$ equals to

$$
\left\{\begin{aligned}
e_{j-1} & \text { if } i=0, \\
e_{j-1}+i \cdot l_{1} & \text { if } 1 \leq i \leq m_{2}-1, \\
e_{j+1} & \text { if } i=m_{2} .
\end{aligned}\right.
$$

We represent the set of intervals given in (1) and (2) to $w_{1}, w_{2}, \cdots, w_{m}$ in a sequential order.

Finally, we consider the frequency of historical data belong to the interval $w_{j}(j=$ $1, \cdots, m)$. If a certain interval $w_{j}$ contains more historical data than that other intervals hold, we do not use information can be inferred from the historical data to construct the fuzzy time series. This causes a decrease in the accuracy of the fuzzy time series constructed by the fuzzy logic. We can improve an accuracy of fuzzy time series by adjusting the frequency of the historical data belong to the interval. Let $f_{i}$ be the frequency of historical data belongs to the interval $w_{i}$ and $f_{\max }$ be 
a maximum frequency subjectively determined by the researcher. If the frequency of the interval $w_{k}$ is greater than $f_{m a x}$, then we repeat to divide $w_{k}$ into several sub-intervals $w_{k}^{(l)}\left(l=1, \cdots, m_{3}\right)$ until $w_{k}^{(l)}$ have the frequency below $f_{\text {max }}$.

We present the intervals obtained by this process as $u_{i}(i=1, \cdots, p)$. Then the intervals $u_{i}$ satisfies the following three conditions at the same time:

(1) The frequency of historical data belong to the interval $u_{i}$ is below $f_{\max }$.

(2) The endpoints of the interval $u_{i}$ are not equal to the historical data.

(3) A center of the interval $u_{j}$ is greater than that of the interval $u_{i}$ if $i \leq j$.

Step 3: Define fuzzy set and fuzzify the crisp values based on the interval proposed in Step 2.

From the intervals $u_{i}(i=1, \cdots, p)$, we define the fuzzy sets $A_{1}, A_{2}, \cdots, A_{p}$ are defined by

$$
\begin{gathered}
A_{1}=1 / u_{1}+0.5 / u_{2}+0 / u_{3}+0 / u_{4}+0 / u_{5}+\cdots+0 / u_{p}, \\
A_{2}=0.5 / u_{1}+1 / u_{2}+0.5 / u_{3}+0 / u_{4}+0 / u_{5}+\cdots+0 / u_{p}, \\
\vdots \\
\vdots \\
A_{i}=0 / u_{1}+\cdots+0.5 / u_{i-1}+1 / u_{i}+0.5 / u_{i+1}+\cdots+0 / u_{p}, \\
\vdots \\
\vdots \\
A_{p}=0 / u_{1}+0 / u_{2}+\cdots+0 / u_{p-2}+0.5 / u_{p-1}+1 / u_{p} .
\end{gathered}
$$

The number that precedes slash symbol "/" is the membership degree of the fuzzy set. If the historical data $x_{t}$ belongs to the interval $u_{i}$ and there is a value represented by a fuzzy set $A_{i}$ in which the maximum membership degree occurs at $u_{i}$, then $x_{t}$ is fuzzified as the fuzzy set $A_{i}$. If we denote the fuzzy data at time $t$ as $A_{i}$, denoted by $F(t)=A_{i}$, then time series consisting of crisp value $\left\{x_{t}: t=1,2, \cdots, n\right\}$ is translated to fuzzy time series $\{F(t): t=1,2, \cdots, n\}$. Let $c_{i}$ be a center of the interval $u_{i}$. The inequality $c_{i}<c_{j}$ for $i<j$ implies that $u_{i}<u_{j}$. Then we define the well-ordered fuzzy sets $A_{i}(i=1, \cdots, p)$ from the well-ordered intervals $u_{i}(i=1, \cdots, p)$. That is, $A_{i}<A_{j}$ if $u_{i}<u_{j}$.

Step 4: Establish the fuzzy logical relationships and the fuzzy logical relationship groups based on the well-ordered fuzzy set $\left\{A_{i}: i=1, \cdots, p\right\}$.

Step 5: Rearrange the fuzzy set using the fuzzy logical relationship.

We denote by the number $r_{i}$ as a maximum value of the set $\left\{\left|i-i_{j}\right|: F(t)=\right.$ $\left.A_{i}, F(t+1)=A_{i_{j}}, j=1, \cdots, k\right\}$ generated by the fuzzy logical relationship about 
$A_{i}$. In the next step, we are going to use an average of the modes of the fuzzy sets in order to obtain the forecasted value of the historical data $x_{t}$ at the time $t$. Since the sample mean is insensitive to outliers, the forecasting accuracy may be affected by the outliers when the value $r_{i}$ is large. Thus, it is reasonable that the researcher adjusts to the value $r_{i}$ to obtain the high forecasting accuracy. For this, we let $r_{\max }$ be the permissible value designed by the researcher. If the fuzzy set given in Step 4 satisfies the relation $r_{i} \geq r_{\max }$, we return back to Step 3 and then repeat the process of dividing the intervals until $r_{i}<r_{\max }$ for each $i$. As like to Step 4, we present the fuzzy sets obtained by this process as $A_{i}\left(i=1, \cdots, p_{1}\right)$ without loss of generality.

Step 6: Predict the historical data using the fuzzy sets.

From the fuzzy sets and the fuzzy logical relationships given in the previous step, we can obtain the forecasted value $\left(\hat{x}_{t}\right)$ of the historical data $x_{t}$ at the time $t$. If $F(t-1)=A_{i}$ and the fuzzy logical relationship group is $A_{i} \rightarrow A_{i_{1}}, A_{i_{2}}, \cdots, A_{i_{q}}$, $q \geq 1$, then the forecasted value $\hat{x}_{t}$ is

$$
\hat{x}_{t}=\frac{1}{q} \sum_{j=1}^{q} c_{i_{j}},
$$

where $c_{i_{j}}$ is the center of the interval $u_{i_{j}}$.

\section{Empirical Studies}

In this section, we present our method to forecast the enrollment of the University of Alabama and TAIFEX introduced in many literatures to show the efficiency of the fuzzy time series. Also, we introduce the new target data KUEXR.

4.1. Forecasting for enrollment The enrollment of the University of Alabama introduced by Song and Chissom([9]) was used in the previous studies of fuzzy time series. This is also used here to show the superiority of the proposed model.

Step 1: According to the actual enrollment of the University of Alabama shown in Table I, we can see that $x_{(1)}=13055$ and $x_{(2)}=19337$ are actual minimum and maximum enrollment, respectively. Now, we let two proper positive numbers $c=55$ and $d=163$. Then we define the universe of discourse $U=[13000,20000]$.

Step 2: The length of the intervals determined by the average-based length of intervals $([3])$ is 300. Since there are no enrollment in the interval [19600,20000], this interval is removed. Hence the $U$ can be divided into equal-length intervals as follows: $w_{i}=[13000+300 \cdot(i-1), 13000+300 \cdot i]$, where $i=1,2, \cdots, 22$. We let 
Table I

Enrollment and fuzzy enrollment

\begin{tabular}{cccc}
\hline Year & Enrollment & $\begin{array}{c}\text { Fuzzy enrollment } \\
\text { based on step 2 }\end{array}$ & $\begin{array}{c}\text { Fuzzy enrollment } \\
\text { based on step 5 }\end{array}$ \\
\hline 1971 & 13055 & $A_{1}$ & $A_{1}$ \\
1972 & 13563 & $A_{2}$ & $A_{2}$ \\
1973 & 13847 & $A_{3}$ & $A_{3}$ \\
1974 & 14696 & $A_{6}$ & $A_{6}$ \\
1975 & 15460 & $A_{10}$ & $A_{14}$ \\
1976 & 15311 & $A_{9}$ & $A_{12}$ \\
1977 & 15603 & $A_{12}$ & $A_{16}$ \\
1978 & 15861 & $A_{13}$ & $A_{17}$ \\
1979 & 16807 & $A_{16}$ & $A_{21}$ \\
1980 & 16919 & $A_{17}$ & $A_{23}$ \\
1981 & 16388 & $A_{15}$ & $A_{19}$ \\
1982 & 15433 & $A_{10}$ & $A_{13}$ \\
1983 & 15497 & $A_{11}$ & $A_{15}$ \\
1984 & 15145 & $A_{8}$ & $A_{9}$ \\
1985 & 15163 & $A_{8}$ & $A_{10}$ \\
1986 & 15984 & $A_{13}$ & $A_{17}$ \\
1987 & 16859 & $A_{16}$ & $A_{22}$ \\
1988 & 18150 & $A_{21}$ & $A_{27}$ \\
1989 & 18970 & $A_{23}$ & $A_{29}$ \\
1990 & 19328 & $A_{25}$ & $A_{32}$ \\
1991 & 19337 & $A_{25}$ & $A_{33}$ \\
1992 & 18876 & $A_{23}$ & $A_{29}$ \\
\hline
\end{tabular}

$f_{\max }=3$. Since the interval $w_{8}$ and $w_{9}$ have the frequency over $f_{\max }$, we repeat to divide into two sub-intervals of the equal length until the sub-intervals have the frequency below $f_{\max }$.

(a) $w_{8}=[15100,15400]$ : The enrollments in 1976, 1984, and 1985 belong to $w_{8}$. Thus, we divide $w_{8}$ into $w_{8}^{(1)}=[15100,15250]$ and $w_{8}^{(2)}=[15250,15400]$.

(b) $w_{9}=[15400,15700]$ : The enrollments in 1795, 1977, 1982, and 1983 belong to $w_{9}$. First, we divide $w_{9}$ into $w_{9}^{(1)}=[15400,15550]$ and $w_{9}^{(2)}=[15550,15700]$. But the enrollments in 1795,1982 and 1983 belong to $w_{9}^{(1)}=[15400,15550]$. Thus, we divide $w_{9}^{(1)}=[15400,15550]$ into $w_{9}^{(1,1)}=[15400,15475]$ and $w_{9}^{(1,2)}=[15475,15550]$. Finally, we get the following intervals:

$$
u_{i}=\left\{\begin{array}{lc}
{[13000+300 \cdot(i-1), 13000+300 \cdot i]} & 1 \leq i \leq 7, \\
{[15100+150 \cdot(i-8), 15100+150 \cdot(i-7)]} & 8 \leq i \leq 9, \\
{[15400+75 \cdot(i-10), 15400+75 \cdot(i-9)]} & 10 \leq i \leq 11, \\
{[15550,15700]} & i=12, \\
{[15700+300 \cdot(i-13), 15700+300 \cdot(i-12)]} & 13 \leq i \leq 25 .
\end{array}\right.
$$


Step 3: Define fuzzy set based on interval in (3) and fuzzify the enrollment. Table I lists the enrollment at the University of Alabama and the corresponding fuzzy enrollment. Fuzzy sets given in Table I provide the fuzzy logical relationships in the left side of Table II.

Step 4: Establish the fuzzy logical relationships and groups. Since the fuzzy logical relationships are $A_{1} \rightarrow A_{2}, A_{2} \rightarrow A_{3}, A_{3} \rightarrow A_{6}, A_{6} \rightarrow A_{10}, A_{10} \rightarrow A_{11}, A_{11} \rightarrow$ $A_{12}, \cdots, A_{25} \rightarrow A_{25}, A_{25} \rightarrow A_{23}$, the fuzzy logical relationship groups look as above Table II.

Step 5: In Table II, we find the fuzzy logical relationship groups having the high variation of fuzzy logical relationships and divide corresponding intervals into subintervals. In this paper we let $r_{\max }=2$. Since $r_{i}$ is greater than $r_{\max }$, we have to re-divide the intervals (3) given in Step 2.

(a) $A_{8} \rightarrow A_{8}, A_{13}$ : Since $r_{8}$ is greater than $r_{\max }$, we have to divide $u_{8}$ into $u_{8}^{(1)}=$ $[15100,15175]$ and $u_{8}^{(2)}=[15175,15250]$. But the all enrollments in the interval $u_{8}$ fall in the interval $u_{8}^{(1)}=[15100,15175]$. Thus, we repeat to divide into two sub-intervals of equal length until the enrollments in the interval $u_{8}$ do not fall in the same sub-interval. We get the resulting intervals as follows: [15100,15137.5], [15137.5, 15156.25], [15156.25, 15175], [15175, 15250].

(b) $A_{10} \rightarrow A_{9}, A_{12}$ : Since $r_{10}$ is greater than $r_{\max }$, the interval $u^{10}=[15400,15475]$

Table II

Fuzzy logical relationship groups (FLRG)

\begin{tabular}{ll}
\hline FLRG based on step 3 & FLRG based on step 5 \\
\hline$A_{1} \rightarrow A_{2}$ & $A_{1} \rightarrow A_{2}$ \\
$A_{2} \rightarrow A_{3}$ & $A_{2} \rightarrow A_{3}$ \\
$A_{3} \rightarrow A_{6}$ & $A_{3} \rightarrow A_{6}$ \\
$A_{6} \rightarrow A_{10}$ & $A_{6} \rightarrow A_{14}$ \\
$A_{8} \rightarrow A_{8}, A_{13}$ & $A_{9} \rightarrow A_{10}$ \\
$A_{9} \rightarrow A_{12}$ & $A_{10} \rightarrow A_{17}$ \\
$A_{10} \rightarrow A_{9}, A_{11}$ & $A_{12} \rightarrow A_{16}$ \\
$A_{11} \rightarrow A_{8}$ & $A_{13} \rightarrow A_{15}$ \\
$A_{12} \rightarrow A_{13}$ & $A_{14} \rightarrow A_{12}$ \\
$A_{13} \rightarrow A_{16}$ & $A_{15} \rightarrow A_{9}$ \\
$A_{15} \rightarrow A_{10}$ & $A_{16} \rightarrow A_{17}$ \\
$A_{16} \rightarrow A_{17}, A_{21}$ & $A_{17} \rightarrow A_{22}$ \\
$A_{17} \rightarrow A_{15}$ & $A_{19} \rightarrow A_{13}$ \\
$A_{21} \rightarrow A_{23}$ & $A_{21} \rightarrow A_{23}$ \\
$A_{23} \rightarrow A_{25}$ & $A_{22} \rightarrow A_{27}$ \\
$A_{25} \rightarrow A_{23}, A_{25}$ & $A_{23} \rightarrow A_{19}, A_{27} \rightarrow A_{29}$ \\
& $A_{29} \rightarrow A_{32}, A_{32} \rightarrow A_{33}$ \\
& $A_{33} \rightarrow A_{29}$ \\
\hline
\end{tabular}


divides into the two sub-intervals $[15400,15437.5]$ and $[15437.5,15475]$.

(c) For $A_{16} \rightarrow A_{17}, A_{21}$ and $A_{25} \rightarrow A_{23}, A_{25}$, we apply the same method above.

Finally, we get the resulting intervals as follows: $u_{i}=[13000+300 \cdot(i-1), 13000+$ $300 \cdot i](i=1,2, \cdots, 7), u_{9}=[15137.5,15156.25], u_{10}=[15156.25,15175], u_{11}=[15175$, $15250], u_{12}=[15250,15400], u_{13}=[15400,15437.5], u_{14}=[15437.5,15475], u_{15}=[15475$, $15550], u_{16}=[15550,15700] u_{17}=[15700,16000], u_{18}=[16000,16300], u_{19}=[16300,16600]$, $u_{20}=[16600,16750], u_{21}=[16750,16825], u_{22}=[16825,16900], u_{i+9}=[13000+300$. $(i-1), 13000+300 \cdot i](i=14,15, \cdots, 21), u_{31}=[19300,19318.75], u_{32}=[19318.75$, $19328.13], u_{33}=[19328.13,19337.5], u_{34}=[19337.5,19375], u_{35}=[19375,19450], u_{36}=[$ 19450,19600 ].

Also, we establish the final fuzzy logical relationships and groups based on the intervals given above in Table II.

Step 6: Defuzzify the forecasting results using the "Centroid". The forecasted enrollments by proposed method and Chen's method([1]) are shown in Table III, respectively.

Table III

Forecasted enrollment

\begin{tabular}{llll}
\hline Year & Actual data & Chen's method & The proposed method \\
\hline 1971 & 13055 & & \\
1972 & 13563 & 14000 & 13450 \\
1973 & 13867 & 14000 & 13750 \\
1974 & 14696 & 14000 & 14650 \\
1975 & 15460 & 15500 & 15456.2 \\
1976 & 15311 & 16000 & 15325 \\
1977 & 15603 & 16000 & 15625 \\
1978 & 15861 & 16000 & 15887.5 \\
1979 & 16807 & 16000 & 16787.5 \\
1980 & 16919 & 16833 & 17050 \\
1981 & 16388 & 16833 & 16450 \\
1982 & 15433 & 16833 & 15418.8 \\
1983 & 15497 & 16000 & 15512.5 \\
1984 & 15145 & 16000 & 15146.8 \\
1985 & 15163 & 16000 & 15165.6 \\
1986 & 15984 & 16000 & 15962.5 \\
1987 & 16859 & 16000 & 16862.5 \\
1988 & 18150 & 16833 & 18250 \\
1989 & 18970 & 19000 & 18850 \\
1990 & 19328 & 19000 & 19323.4 \\
1991 & 19337 & 19000 & 19332.8 \\
1992 & 18876 & 19000 & 18850 \\
\hline
\end{tabular}


Fig.I shows the actual and forecasted data. From Fig.I, we see that our method reflects the fluctuation of historical data.

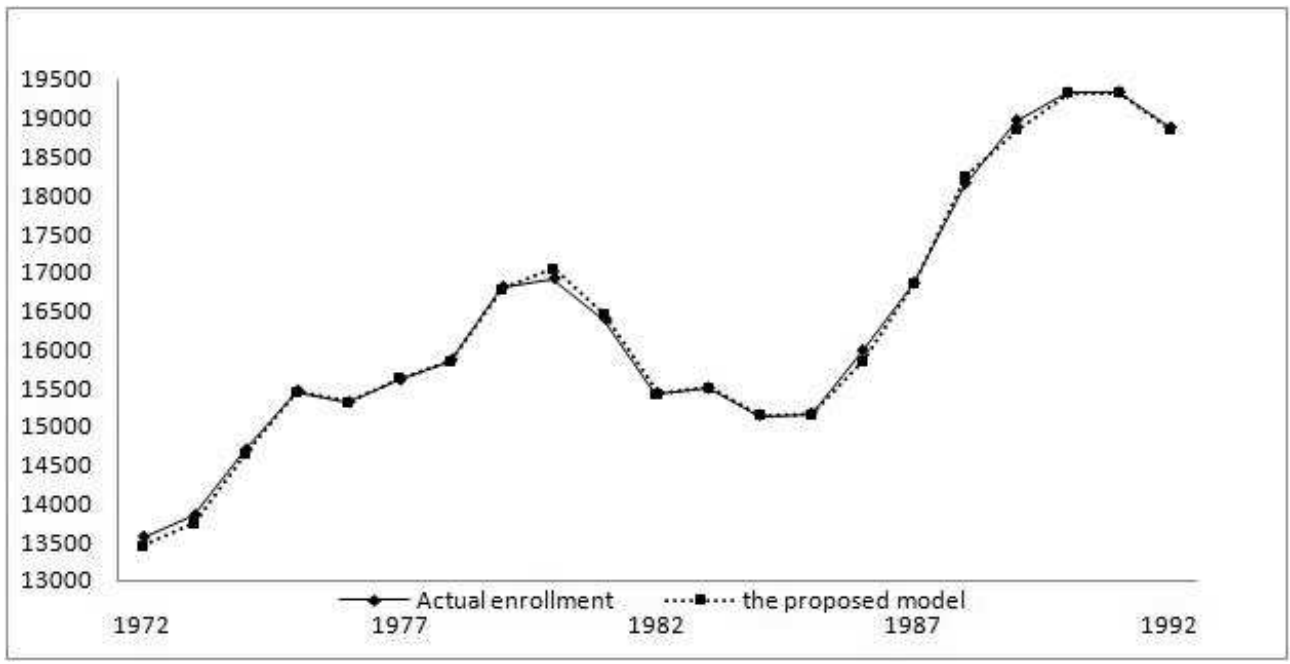

Fig.I. The actual and forecasted enrollment

4.2. Forecasting for TAIFEX TAIFEX data set introduced to Huarng([4]) has been used as the forecasting target for many authors studying the fuzzy time series. The proposed method gets higher the forecasting accuracy and easier calculation than the other methods using the TAIFEX.

Step 1: According to the actual TAIFEX shown in Table IV, we can see that $x_{(1)}=6200$ and $x_{(2)}=7560$ are actual minimum and maximum data, respectively. Now, we let two proper positive numbers $c=50$ and $d=40$. Then we define the universe of discourse $U=[6150,7600]$.

Step 2: The length of the intervals using average-based length is 40 (Huarng $([3]))$. Since there are no actual data in the interval $[6150,6190]$, this interval is removed. Hence the $U$ can be divided into equal-length intervals as follows: $v_{i}=$ $[6190+40 \cdot(i-1), 6190+40 \cdot i]$, for $i=1,2,3, \cdots, 34$, and $v_{35}=[7550,7600]$. We let $f_{\max }=3$. Then we obtain 53 intervals having the frequency of historical data below $f_{\text {max }} .5$

Step 3-5: We let $r_{\max }=3$. Then we obtain 73 intervals having $r_{i}$ below $r_{\max }$. Since the methods are similar to those in the A. Forecasting for enrollment, for the sake of simplicity, we omit the processes of establishing fuzzy sets and fuzzy logical relationship groups. 
Step 6: Defuzzify the forecasting results using the "Centroid". The forecasted TAIFEX by proposed method and existing methods are shown in Table IV.

Table IV

Forecasted TAIFEX

\begin{tabular}{llllll}
\hline date & actual data & $\begin{array}{l}\text { Chen's } \\
\text { method }\end{array}$ & $\begin{array}{l}\text { Huarng's } \\
\text { method }\end{array}$ & $\begin{array}{l}\text { Lee et al.'s } \\
\text { method([6]) }\end{array}$ & $\begin{array}{l}\text { The Proposed } \\
\text { method }\end{array}$ \\
\hline $8 / 04$ & 7560 & 7450 & 7450 & & 7559.375 \\
$8 / 05$ & 7487 & 7450 & 7450 & & 7490 \\
$8 / 06$ & 7462 & 7500 & 7500 & & 7450 \\
$8 / 07$ & 7515 & 7500 & 7500 & & 7530 \\
$8 / 10$ & 7365 & 7450 & 7450 & & 7370 \\
$8 / 11$ & 7360 & 7300 & 7300 & & 7350 \\
$8 / 12$ & 7330 & 7300 & 7300 & 7329 & 7350 \\
$8 / 13$ & 7291 & 7300 & 7300 & 7289.5 & 7296.667 \\
$8 / 14$ & 7320 & 7183.33 & 7183.33 & 7329 & 7330 \\
$8 / 15$ & 7300 & 7300 & 7300 & 7289.5 & 7296.667 \\
$8 / 17$ & 7219 & 7300 & 7300 & 7215 & 7218.751 \\
$8 / 18$ & 7220 & 7183.33 & 7100 & 7215 & 7219.861 \\
$8 / 19$ & 7285 & 7183.33 & 7300 & 7289.5 & 7285 \\
$8 / 20$ & 7274 & 7183.33 & 7183.33 & 7289.5 & 7275 \\
$8 / 21$ & 7225 & 7183.33 & 7100 & 7215 & 7225 \\
$8 / 24$ & 6955 & 7183.33 & 7100 & 6949.5 & 6970 \\
$8 / 25$ & 6949 & 6850 & 6850 & 6949.5 & 6921.667 \\
$8 / 26$ & 6790 & 6850 & 6850 & 6796 & 6790 \\
$8 / 27$ & 6835 & 6775 & 6775 & 6848 & 6833.333 \\
$8 / 28$ & 6695 & 6850 & 6750 & 6698.5 & 6695 \\
$8 / 29$ & 6728 & 6750 & 6750 & 6726 & 6725.625 \\
$8 / 31$ & 6566 & 6775 & 6650 & 6569.5 & 6570 \\
$9 / 01$ & 6409 & 6450 & 6450 & 6417 & 6410 \\
$9 / 02$ & 6430 & 6450 & 6550 & 6417 & 6430 \\
$9 / 03$ & 6200 & 6450 & 6350 & 6205 & 6210 \\
$9 / 04$ & 6403.2 & 6450 & 6450 & 6417 & 6396.667 \\
$9 / 05$ & 6697.5 & 6450 & 6550 & 6698.5 & 6695 \\
$9 / 07$ & 6722.3 & 6450 & 6750 & 6726 & 6725.625 \\
$9 / 08$ & 6859.4 & 6750 & 6850 & 6848 & 6857.5 \\
$9 / 09$ & 6769.6 & 6775 & 6750 & 6763 & 6766.667 \\
$9 / 10$ & 6709.75 & 6850 & 6650 & 6726 & 6705 \\
$9 / 11$ & 6726.5 & 6775 & 6775 & 6726 & 6726.25 \\
$9 / 14$ & 6774.55 & 6775 & 6775 & 6763 & 6773.333 \\
$9 / 15$ & 6762 & 6775 & 6775 & 6763 & 6756.667 \\
$9 / 16$ & 6952.75 & 6775 & 6850 & 6949.5 & 6970 \\
$9 / 17$ & 6906 & 6775 & 6850 & 6904.5 & 6921.667 \\
$9 / 18$ & 6842 & 6850 & 6850 & 6848 & 6842.222 \\
$9 / 19$ & 7039 & 6850 & 6850 & 7064 & 7050 \\
$9 / 21$ & 6861 & 6850 & 6850 & 6848 & 6865 \\
$9 / 22$ & 6926 & 6850 & 6850 & 6904.5 & 6920 \\
$9 / 23$ & 6852 & 6850 & 6850 & 6848 & 6852.5 \\
$9 / 24$ & 6890 & 6850 & 6850 & 6904.5 & 6890 \\
$9 / 25$ & 6871 & 6850 & 6850 & 6848 & 6873.333 \\
$9 / 28$ & 6840 & 6850 & 6750 & 6848 & 6840 \\
$9 / 29$ & 6806 & 6850 & 6850 & 6796 & 6816.667 \\
$9 / 30$ & 6787 & 6850 & 6750 & 6796 & 6790 \\
\hline & & & & &
\end{tabular}




\subsection{Forecasting for KRW/USD exchange rate}

Table V

Forecasted KUEXR

\begin{tabular}{|c|c|c|c|c|}
\hline date(year) & month) & actual data & Liu's method & The Proposed method \\
\hline $06 / 01$ & & 983.76 & & \\
\hline $06 / 02$ & & 969.96 & 969 & 969.8333 \\
\hline $06 / 03$ & & 975.17 & 979 & 979 \\
\hline $06 / 04$ & & 952.92 & 969 & 969.8333 \\
\hline $06 / 05$ & & 941.21 & 959 & 950.25 \\
\hline $06 / 06$ & & 955.28 & 939 & 945.6667 \\
\hline $06 / 07$ & & 950.56 & 944 & 943.375 \\
\hline $06 / 08$ & & 961.02 & 959 & 950.25 \\
\hline $06 / 09$ & & 952.77 & 944 & 943.375 \\
\hline $06 / 10$ & & 954.17 & 959 & 950.25 \\
\hline $06 / 11$ & & 935.52 & 944 & 943.375 \\
\hline $06 / 12$ & & 925.08 & 939 & 929 \\
\hline $07 / 01$ & & 936.90 & 929 & 927.75 \\
\hline $07 / 02$ & & 937.17 & 939 & 939.625 \\
\hline $07 / 03$ & & 943.23 & 939 & 939.625 \\
\hline $07 / 04$ & & 930.95 & 939 & 945.6667 \\
\hline $07 / 05$ & & 927.39 & 929 & 929 \\
\hline $07 / 06$ & & 928.16 & 929 & 927.75 \\
\hline $07 / 07$ & & 918.45 & 929 & 927.75 \\
\hline $07 / 08$ & & 934.92 & 929 & 928.5833 \\
\hline $07 / 09$ & & 930.89 & 939 & 929 \\
\hline $07 / 10$ & & 914.81 & 929 & 929 \\
\hline $07 / 11$ & & 918.11 & 929 & 928.5833 \\
\hline $07 / 12$ & & 930.76 & 929 & 928.5833 \\
\hline $08 / 01$ & & 942.72 & 929 & 929 \\
\hline $08 / 02$ & & 944.42 & 939 & 945.6667 \\
\hline $08 / 03$ & & 982.51 & 959 & 979 \\
\hline $08 / 04$ & & 987.24 & 969 & 969.8333 \\
\hline $08 / 05$ & & 1038.21 & 1039 & 1039 \\
\hline $08 / 06$ & & 1031.07 & 1029 & 1029 \\
\hline $08 / 07$ & & 1018.18 & 1019 & 1019 \\
\hline $08 / 08$ & & 1047.11 & 1049 & 1049 \\
\hline $08 / 09$ & & 1136.64 & 1139 & 1137.125 \\
\hline $08 / 10$ & & 1326.85 & 1202.333 & 1329 \\
\hline $08 / 11$ & & 1400.81 & 1399 & 1399 \\
\hline $08 / 12$ & & 1368.80 & 1369 & 1369 \\
\hline $09 / 01$ & & 1354.68 & 1359 & 1359 \\
\hline $09 / 02$ & & 1440.19 & 1439 & 1439 \\
\hline $09 / 03$ & & 1453.35 & 1449 & 1449 \\
\hline $09 / 04$ & & 1336.28 & 1339 & 1339 \\
\hline $09 / 05$ & & 1255.62 & 1259 & 1259 \\
\hline $09 / 06$ & & 1262.28 & 1209 & 1249 \\
\hline $09 / 07$ & & 1262.96 & 1209 & 1249 \\
\hline $09 / 08$ & & 1239.69 & 1209 & 1249 \\
\hline $09 / 09$ & & 1215.00 & 1219 & 1219 \\
\hline $09 / 10$ & & 1174.80 & 1179 & 1179 \\
\hline $09 / 11$ & & 1163.18 & 1159 & 1161.5 \\
\hline $09 / 12$ & & 1166.13 & 1182.333 & 1165.25 \\
\hline $10 / 01$ & & 1138.77 & 1179 & 1138.375 \\
\hline $10 / 02$ & & 1156.83 & 1202.333 & 1156.5 \\
\hline $10 / 03$ & & 1136.11 & 1182.333 & 1135.25 \\
\hline $10 / 04$ & & 1115.71 & 1202.333 & 1119 \\
\hline $10 / 05$ & & 1168.41 & 1169 & 1167.75 \\
\hline $10 / 06$ & & 1214.02 & 1179 & 1219 \\
\hline
\end{tabular}

In this section, we investigate KRW/USD average exchange rate per month(KUEXR) to ensure that the proposed method is superior to the other methods.

Step 1: According to the actual KUEXR shown in Table V, we can see that $x_{(1)}=$ 914.81 and $x_{(2)}=1453.35$ are actual minimum and maximum data, respectively. 
Then we define the universe of discourse $U=[914,1454]$.

Step 2: The length of the intervals using average-based length is 10(Huarng ([3])). Also, we let $f_{\max }=4$. Then we obtain 33 intervals having the frequency of historical data below $f_{\max }$.

Step 3-5: We let $r_{\max }=4$. Then we obtain 41 intervals having $r_{i}$ below $r_{\max }$. For the sake of simplicity, we omit the processes of establishing fuzzy sets and fuzzy logical relationship groups.

Step 6: Defuzzify the forecasting results using the "Centroid". The forecasted KUEXR by proposed method and Liu([7]) are shown in Table V.

Fig.II shows the actual and forecasted data. From Fig.II we see that our method reflects the fluctuation of historical data.

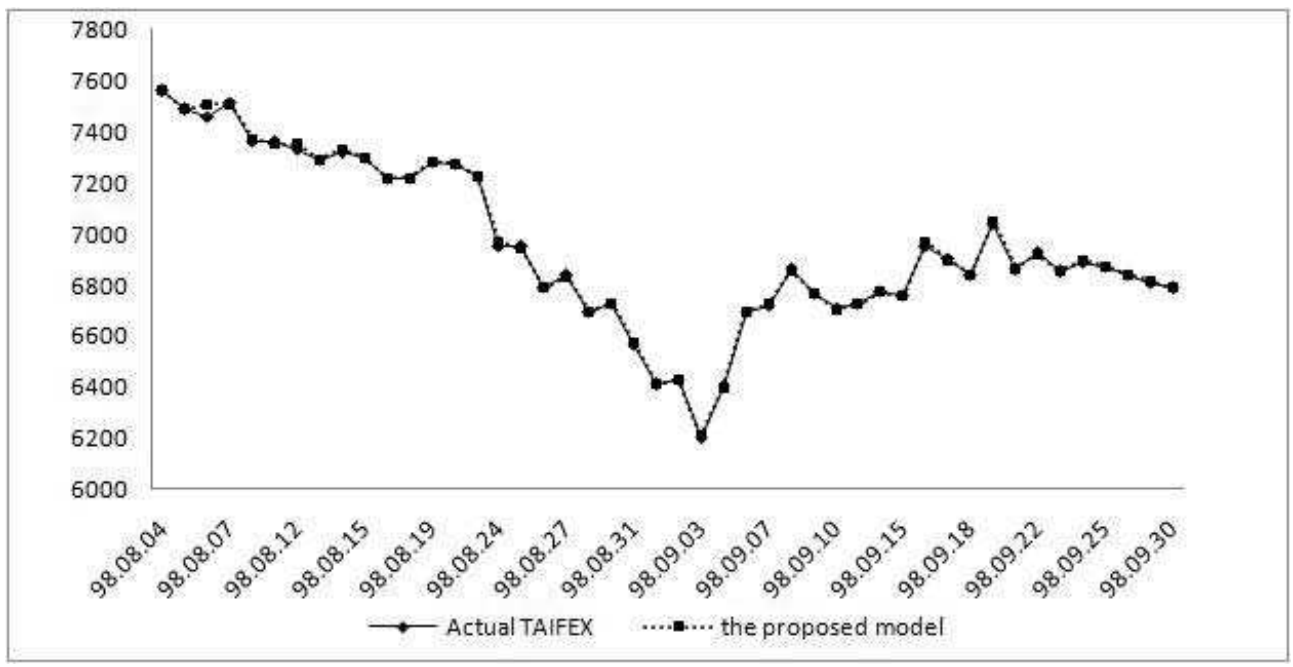

Fig.II. The actual and forecasted TAIFEX

\section{The Forcasting Accuracy Validation}

In this section, we compare a performance of proposed method with various fuzzy time series constructed by many authors.

The goal of forecasting, which is the process of estimation in unknown situations, is to be as accurate as possible. For this purpose, we consider a performance measure providing forecasting error, which is the difference between the actual value $\left(x_{t}\right)$ and the forecasted value $\left(\hat{x}_{t}\right)$. The Mean Square Error(MSE) and Mean Absolute Percentage Error(MAPE) are used to measure the forecasting accuracy: 


$$
\begin{aligned}
\text { MSE } & =\frac{1}{n} \sum_{t=1}^{n}\left(x_{t}-\hat{x}_{t}\right)^{2}, \\
\text { MAPE } & =\frac{1}{n} \sum_{t=1}^{n} \frac{\left|x_{t}-\hat{x}_{t}\right|}{\left|x_{t}\right|} .
\end{aligned}
$$

Table VI shows that the proposed method's forecasting accuracy is superior to existing methods. The MSE of Liu's method is 108097 but that of the proposed method is 4287. Also, the MAPE of Liu's method is 1.33 but that of the proposed method is 0.0028 .

Table VI

The forecasting accuracy for enrollment

\begin{tabular}{llllll}
\hline Index & $\begin{array}{l}\text { Chen's } \\
\text { method }\end{array}$ & $\begin{array}{l}\text { Huarng's } \\
\text { method }\end{array}$ & $\begin{array}{l}\text { Cheng's } \\
\text { method }\end{array}$ & $\begin{array}{l}\text { Liu's } \\
\text { method }\end{array}$ & $\begin{array}{l}\text { The proposed } \\
\text { method }\end{array}$ \\
\hline MSE & 407507 & 226611 & 191844 & 108097 & 4287 \\
MAPE $(\%)$ & 3.11 & 2.45 & 2.09 & 1.33 & 0.0028 \\
\hline
\end{tabular}

Now, we consider the forecasting accuracy for the TAIFEX given by Chen([1]), Huarng $([4])$ and Lee et al.([5,6]). From Table VII, we can see that the MSE and MAPE of the proposed method are obviously smaller than those of other methods.

Table VII

The forecasting accuracy for TAIFEX

\begin{tabular}{llllll}
\hline Index & $\begin{array}{l}\text { Chen's } \\
\text { method }\end{array}$ & $\begin{array}{l}\text { Huarng's } \\
\text { method }\end{array}$ & $\begin{array}{l}\text { Lee et al.'s } \\
\text { method([5]) }\end{array}$ & $\begin{array}{l}\text { Lee et al.'s } \\
\operatorname{method}([6])\end{array}$ & $\begin{array}{l}\text { The proposed } \\
\text { method }\end{array}$ \\
\hline MSE & 9668.97 & 5389.3 & 1364.56 & 105.02 & 67.71 \\
MAPE(\%) & 1.051 & 0.857 & 0.434 & 0.119 & 0.00077 \\
\hline
\end{tabular}

Table VIII shows that MSE and MAPE of the proposed method for our new target data KUEXR is obviously smaller than those of the Liu's method ([7]). The MSE of Liu's method is 756.9311 but that of the proposed method is 50.50642 .

Table VIII

The forecasting accuracy for KUEXR

\begin{tabular}{lll}
\hline Index & Liu's method & The proposed method \\
\hline MSE & 756.4894 & 50.50642 \\
MAPE(\%) & 0.013582 & 0.005156 \\
\hline
\end{tabular}

We considered frequency of historical data belongs to one interval(step 2), and distance of the fuzzy logical relationship(step 5) at the same time. So we could reflect the fluctuation of historical data, which resulted in great performance. 


\section{Conclusions}

We proposed a new method of fuzzy time series for improving the forecasting accuracy. In this paper, we considered the frequency of historical data belong to some fuzzy set and the distance between fuzzy sets in the fuzzy logical relationship group. The frequency and distance played important roles to improve the forecasting accuracy and to reflect the fluctuation of historical data. Empirical studies illustrated that the proposed method's forecasting accuracy is superior to existing methods.

\section{REFERENCES}

1. Chen, S.M.: Forecasting enrollments based on fuzzy time series. Fuzzy Sets and Systems 81 (1996), 311-319.

2. Cheng, C.H., Chen, T.L., Teoh, H.J. \& Chiang, C.H.: Fuzzy time-series based on adaptive expectation model for TAIEX forecasting. Expert Systems with Applications 34 (2008), 1126-1132.

3. Huarng, K.: Effective lengths of intervals to improve forecasting in fuzzy time series. Fuzzy Sets and Systems 123 (2001a), 155-162.

4. _ : Heuristic models of fuzzy time series for forecasting. Fuzzy Sets and System $\mathbf{1 2 3}$ (2001b), 369-386.

5. Lee, L.W., Wang, L.H., Chen, S.M. \& Leu, Y.H.: Handling forecasting problems based on two-factors high-order fuzzy time series. IEEE Transactions on Fuzzy Systems 14 (2006), 468-477.

6. Lee, L.W., Wang, L.H. \& Chen, S.M.: Temperature prediction and TAIFEX forecasting based on high-order fuzzy logical relationships and genetic simulated annealing techniques. Expert Systems with Applications 34 (2008), 328-336.

7. Liu, H.T.: An improved fuzzy times forecasting method using trapezoidal fuzzy numbers. Fuzzy Optimization Decision Making 6 (2007), 63-80.

8. Song, Q. \& Chissom, B.S.: Forecasting enrollments with fuzzy time series-part I. Fuzzy Sets and Systems $\mathbf{5 4}$ (1993a), 1-10.

9. _ : Forecasting enrollments with fuzzy time series-part II. Fuzzy Sets and Systems 62 (1993a), 1-8

a Department of Mathematics, Yonsei University, Seoul 120-749, Korea

Email address: pinkjhy@hanmail.net

${ }^{\mathrm{b}}$ School of Economics, Yonsei University, Seoul 120-749, Korea

Email address: jin91350yonsei.ac.kr

${ }^{c}$ School of Liberal Arts and Science, Korea Aerospace University, Goyang 412-791, KOREA

Email address: shchoi@kau.ac.kr 